\title{
Should people at low risk of cardiovascular disease take a statin?
}

The conclusion and summary box of this Analysis article by Abramson and colleagues (BMJ 2013;347:f6123, doi:10.1136/ bmj.f6123) stated that side effects of statins occur in about $18-20 \%$ of patients. The authors withdraw this statement. Although it was based on statements in the referenced observational study by Zhang and colleagues, that "the rate of reported statin-related events to statins was nearly $18 \%, " 1$ the article did not reflect necessary caveats and did not take sufficient account of the uncontrolled nature of the study.

Zhang et al observed that the rate of statin related events found in their study (18\%) was "substantially higher than the $5 \%$ to $10 \%$ usually described in randomized, placebo-controlled, clinical trials." Two caveats must be considered. As Zhang et al point out, the rate of statin related events reported in their study was uncontrolled and therefore may be inflated because events attributed to statins might have occurred in a placebo group as well. In addition, although Zhang et al do not make this point, the $5-10 \%$ rate quoted by Zhang et al as having been observed in randomised trials was, in many cases, similar in both active and placebo groups.

The exact rate of statin related adverse events in people at low risk of cardiovascular disease remains uncertain. Clinical trials may underestimate the frequency of statin related adverse events because of patient selection, exclusion of older patients and those with comorbid conditions or potential drug interactions, under-representation of women, and selection bias created by willingness to participate in a clinical trial. In addition, when compared with the full clinical study reports, published accounts of clinical trials in medical journals report only a minority of adverse events. ${ }^{2}$ Access to the full data from the trials of statins would help to determine the comparative rates of serious adverse events in statin and control groups but probably would not help to determine the frequency of less than serious adverse events.
The authors also mistakenly reported that Zhang et al found that " $18 \%$ of statin treated patients had discontinued therapy (at least temporarily) because of statin related events." The correct interpretation of the data, as confirmed to The BMJ by Zhang et al, is as follows. Based on review of structured electronic medical record categories and automated review of unstructured narratives from follow-up visits of 107835 patients over eight years, 18778 of all study patients $(17.4 \%)$ had a statin related event documented during the study. Among those who experienced a statin related event, only $59.2 \%$ had statin therapy discontinued at least temporarily. However, because of possible miscategorisation resulting from the limited options in the electronic medical record for recording reasons for discontinuation of statin therapy, Zhang et al concluded that "as many as $87 \%$ " of these discontinuations could have been due to statin-related events. This equates to up to $9 \%$ of the study population having possibly discontinued statin therapy as a consequence of statin related events, rather than the $18 \%$ cited.

The primary finding of Abramson and colleague's article - that the Cholesterol Treatment Trialists' data failed to show that statins reduced the overall risk of mortality among people with $<20 \%$ risk of cardiovascular disease over the next 10 years-was not challenged in the process of communication about this correction.

1 Zhang H, Plutzky J, Skentzos S, Morrison F, Mar P, Shubina M, et al. Discontinuation of statins in routine care settings. Ann Intern Med 2013;158:526-34.

2 Wieseler B, Wolfram N, McGauran N, Kerekes MF, Vervolgyi V, et al. Completeness of reporting of patient-relevant clinical trial outcomes: comparison of unpublished clinical study reports with publicly available data. PLoS Med 2013;10:e1001526

Cite this as: BMJ 2014;348:g3329

๑ BMJ Publishing Group Ltd 2014 